\title{
TRABAJO DOCENTE E INCLUSIÓN EDUCATIVA EN TIEMPOS DE LA ASIGNACIÓN UNIVERSAL POR HIJO: POSICIONES, EXPERIENCIAS Y SENTIDOS EN DISPUTA
}

\author{
Por Alejandro Vassiliades* \\ Universidad Nacional de La Plata - CONICET, Argentina. \\ alevassiliades@gmail.com
}

Recibido: 20/09/2016 Aceptado: 31/10/2016

\begin{abstract}
Resumen
Este artículo se propone analizar los modos en que sujetos docentes construyen sentidos acerca su trabajo en la producción de iniciativas institucionales ligadas a la tarea cotidiana con alumnos que viven en condiciones de pobreza. Para ello, aborda la implementación de experiencias pedagógicas en tres escuelas primarias públicas en la provincia de Buenos Aires, y cómo ellas procesan un conjunto de significados acerca de la inclusión educativa puestos en circulación por un conjunto de políticas docentes desarrolladas entre 2003 y 2015. Alrededor de la mitad de las familias de los alumnos de estas escuelas recibe la Asignación Universal por Hijo, política que establece el cumplimiento de la obligatoriedad escolar como requisito para percibir esta prestación. Así, este trabajo se propone analizar los sentidos sobre la inclusión que se entraman en la construcción de posiciones docentes en territorios de la desigualdad en el contexto de implementación de una política que impulsa el acceso y una asistencia sostenida por parte de los estudiantes.
\end{abstract}

Doctor en Educación (UBA). Investigador Asistente del Consejo Nacional de Investigaciones Científicas y Técnicas (CONICET) con sede en el Instituto de Investigaciones en Humanidades y Ciencias Sociales (CONICET-UNLP). Jefe de Trabajos Prácticos de la cátedra "Educación II" (FFyL-UBA), y de la cátedra "Historia de la Educación Argentina y Latinoamericana" (FaHCE-UNLP). Integrante de la Red Latinoamericana de Estudios sobre el Trabajo Docente (Red ESTRADO). 
Palabra Clave Trabajo docente - Posición docente - Inclusión educativa - Política educativa - Análisis del discurso.

\section{Abstract}

This article analyses the ways in which teachers build senses about their work in the production of institutional initiatives linked to their everyday task with students that live in poverty. With that aim, it tackles the implementation of pedagogical experiences in three primary schools in the province of Buenos Aires, and how they process a group of meanings about educational inclusion put into circulation by educational policies between 2003 and 2015. Around half of the schools students' families receive the Universal Child Allowance, a policy that establishes the fulfillment of compulsory schooling as a requirement to perceive this provision. This article intends to analyse the meanings about inclusion that are involved in the building of teaching positions in territories of inequality in the context of the implementation of a policy that encourages school attendance by students.

Keywords Teaching work - Teaching position - Educational inclusion - Educational policy - Discourse analysis.

\section{Introducción: sobre el análisis de los discursos acerca de la inclusión educativa}

La inclusión parece haberse tornado una nota central del discurso pedagógico de los últimos años en Argentina. Convocada recurrentemente como uno de los significantes centrales de las políticas educativas implementadas entre 2003 y 2015, su creciente presencia en la agenda educativa constituye un aspecto que lleva a plantear el interrogante referido a cómo se procesan sus sentidos en las instituciones educativas.

Enmarcado en una perspectiva posfundacional, crítica de cualquier planteo que ancle un determinado significante a un fundamento último, el trabajo de Dussel (2004) plantea dos señalamientos nodales para el abordaje de la cuestión de la inclusión. Por un lado, apunta que este significante no posee un significado fijo y definitivo, y que los sentidos a los que se asocia son inestables e históricamente variables. Por otro lado, argumenta que inclusión y exclusión son parte de un mismo proceso, más que términos opuestos, y que entonces la mirada de las dinámicas referidas a la exclusión de determinados grupos sociales o pautas culturales debe dirigirse hacia el modo en que en diversos momentos históricos se ha concebido la inclusión. Esta perspectiva asume que la inclusión y la exclusión se entraman en un vínculo relacional, y que los modos en que cada uno de estos términos se plantearon implicaron fronteras móviles y efectos en los sentidos que iba asumiendo el otro concepto de este par. 
Una consecuencia de este enfoque es que la inclusión no tiene un único sentido. Como significante en disputa, la tarea que sobreviene es la de dar cuenta de los sentidos múltiples y variables que se asociaron a ella, y los modos en que ellos se insertaron en discursos acerca del trabajo docente y la escolarización. Por ello, este artículo se sitúa en la pregunta referida a los sentidos que se construyeron en torno de la inclusión. Para emprender esta tarea, y sobre la base de una investigación realizada en la provincia de Buenos Aires (1) este artículo explorará las posibilidades de analizar el problema de la inclusión desde el abordaje de las posiciones docentes que se construyen en territorios surcados por la desigualdad social. Para hacerlo, indagará acerca de los discursos que se construyen en torno de la docencia, asumiéndolos como un conjunto de procesos vinculados la producción y circulación de sentidos para establecer significaciones provisorias acerca de una porción de lo Social (Laclau, 1993) referida, en este caso, al trabajo de enseñar.

Estas producciones de sentido poseen efectos en la construcción de regulaciones sobre el trabajo docente, es decir, en las reglas, sentidos y formas de conocimiento acerca del desarrollo de esta tarea. En este marco, la construcción de discursos acerca del trabajo de enseñar no constituye un proceso alejado de la cotidianeidad de las prácticas sino que se articula con los modos en que los docentes piensan e interpretan su trabajo, se interrogan sobre él, formulan problemas y ensayan iniciativas pedagógicas tendientes a su resolución (Popkewitz, 2000; Southwell, 2009).

Para dar cuenta de ese proceso, una de las categorías teóricas centrales en la investigación realizada ha sido la de posición docente. Construido en el marco de una línea de trabajo desarrollada por Myriam Southwell (2009, 2012), en la que se situó la producción de una tesis de doctorado bajo su dirección (Vassiliades, 2012), el concepto de posición docente se refiere a los modos múltiples en que los maestros y profesores asumen, viven y desarrollan el trabajo de enseñar (inasibles desde las ideas de rol o función, ligadas al desempeño de un papel preestablecido), y los problemas y resoluciones que se plantean en torno de ella, que se reformulan y actualizan dinámicamente en la cotidianeidad del desempeño de la tarea en las instituciones escolares.

La categoría posición docente deviene de la noción de posiciones de sujeto (Laclau, 1993) en tanto subjetividades que no puede reducirse a una localización específica en el seno de una totalidad, en tanto las conciencias no son absolutas y las identidades devienen de algo constitutivamente ajeno y son siempre un vacío imposible de ser colmado. En este sentido, es el discurso el que constituye las posiciones de sujeto y no es el individuo el que origina el discurso (Buenfil Burgos, 2007). Toda posición de sujeto es entonces una posición discursiva en tanto participa del carácter abierto de todo discurso y no logra ser fijada totalmente en un sistema cerrado de diferencias. La fijación temporaria de una identidad remite al proceso de identificación como 
un proceso de sutura, un punto de encuentro entre, por un lado, discursos y prácticas que intentan interpelar, que nos hablan o ubican como sujetos sociales de discursos particulares y, por el otro lado, los procesos que producen subjetividades, que construyen sujetos pasibles de ser "dichos" o "nombrados". En este sentido, las identidades son puntos de adhesión temporaria a las posiciones subjetivas que las prácticas discursivas configuran y que articulan al sujeto en el flujo del discurso.

La posición docente, como parte de los procesos de circulación de discursos, involucra diversos sentidos acerca de cómo desarrollar el trabajo de transmisión cultural y qué relación establecer con el saber (Southwell, 2009), qué vínculos de autoridad establecer con las nuevas generaciones, el desarrollo de formas de sensibilidad respecto de lo que les sucede, las consideraciones ético-políticas acerca del desempeño del oficio, y los modos en que los sentidos históricos y contemporáneos sobre el trabajo docente se articulan en las prácticas de maestros y profesores (Southwell y Vassiliades, 2014). Desde estas dimensiones, resulta posible aproximarse a los modos en que los docentes procesan los sentidos en torno de la inclusión que son puestos a circular por el discurso pedagógico oficial, como parte del proceso relativo a la construcción de una posición docente.

Estos procesos de circulación de sentido pueden ser abordados desde la consideración de las políticas docentes como procesos sociales de significación, en los que se intenta fijar de de modos diversos a la docencia como posición de sujeto a determinados sentidos, en el marco de diferentes enunciaciones y significados en disputa (Southwell, 2007), y para lo cual se intervienen ampliamente diversos aspectos normativos y organizativos de la formación y el trabajo de maestros y profesores. En tanto intervenciones discursivas, dichas políticas tienen un carácter eminentemente político, conflictivo y asociado a las luchas por lograr una fijación -siempre precaria- de significados ligados a la docencia.

En este marco, este trabajo se propone ofrecer un análisis de los modos en que sujetos docentes construyen sentidos acerca su trabajo en la producción de iniciativas institucionales ligadas a la tarea cotidiana con alumnos que viven en condiciones de pobreza. Para ello, aborda la implementación de iniciativas pedagógicas en tres escuelas primarias públicas situadas en las afueras de la Ciudad de La Plata y del partido de San Fernando, en la provincia de Buenos Aires, y cómo ellas procesan un conjunto de significados acerca de la inclusión educativa puestos en circulación por un conjunto de políticas docentes desarrolladas entre 2003 y 2015 . Alrededor de la mitad de las familias de los alumnos de estas escuelas recibe la Asignación Universal por Hijo, política que establece el cumplimiento de la obligatoriedad escolar como requisito para percibir esta prestación. Así, este trabajo se propone analizar los sentidos sobre la inclusión que se entraman en la construcción de posiciones 
Revista de la Escuela de Ciencias de la Educación, año 13, nRo. 12, vol. 1, enero a junio de 2017. Páginas 7996. ISSN 1851-6297 - ISSN 2362-3349 (EN LiNEA). Trabajo docente e inclusión educativa en tiempos de la asignación universal por hijo: posiciones, experiencias y sentidos en disputa. ALEJANDRO VASSILIADES

docentes en territorios de la desigualdad en el contexto de implementación de una política que impulsa el acceso y una asistencia sostenida por parte de los estudiantes.

\section{Redefinir el formato institucional: la inclusión ligada a recuperar y con-}

\section{solidar la tarea específicamente "escolar" de los estudiantes}

El trabajo de campo, realizado en tres escuelas públicas de nivel primario en la provincia de Buenos Aires, tuvo el objetivo de analizar la construcción de posiciones docentes en territorios surcados por la desigualdad social. Para ello, la indagación se dirigió a describir, analizar e interpretar la producción y circulación de discursos que configuran posiciones docentes, indagando el modo en que ellos constituyen sentidos, reglas y pautas que organizan el trabajo de enseñar. Las instituciones seleccionadas tienen como característica común el trabajar con estudiantes que en su enorme mayoría habitan hogares con Necesidades Básicas Insatisfechas -en situaciones de hacinamiento, en viviendas precarias y sin servicios básicos-, y que hubieran desarrollado alguna iniciativa pedagógica específica. La construcción de datos empíricos se llevó a cabo a través de entrevistas en profundidad a docentes y directivos y de observaciones del transcurrir cotidiano en las escuelas mencionadas.

El objetivo fue el relevamiento de los discursos -entendiendo por ellos no sólo lo que los sujetos docentes dicen sino cómo ellos "son hablados" por estas configuraciones de sentido- y la reconstrucción de los significados y sentidos que los sujetos docentes construyen y asignan a su trabajo. La investigación adoptó un abordaje de corte cualitativo-interpretativo en el que las aproximaciones a las posiciones docentes asumen que éstas no tienen un anclaje individual distinguido de aquello social a lo que aspiran a dotar de sentido. La posición docente no es entonces una disposición particular de un docente sino una construcción que deviene de la circulación social de discursos, que antecede y excede esa posición.

Las escuelas comparten una situación de descenso matricular en los años previos a la implementación de los proyectos pedagógicos en 2010. Se trata de instituciones cuyos alumnos provienen de asentamientos cercanos y que son hijos/as de padres desocupados o changarines y cartoneros, en muchos casos sin acceso a cubrir las necesidades básicas y que residen en asentamientos cercanos a la institución. La mayoría de las familias de los alumnos está atravesada por situaciones de inestabilidad o precariedad habitacional, no accediendo en algunos casos a los servicios básicos indispensables. Se trata de una población que en gran medida vive en asentamientos o villas cercanas y que proviene del Noroeste y Noreste de la Argentina. Un número menor de familias proviene de países limítrofes, primordialmente de Paraguay y Perú.

Alrededor de la mitad de las familias de la población estudiantil de cada escuela recibe la Asignación Universal por Hijo (AUH). Ella constituye una 
política del Estado Nacional que viene siendo implementada desde noviembre 2009 por parte de la Administración Nacional de la Seguridad Social (ANSES), y consiste en el otorgamiento de una prestación no contributiva similar a la que reciben los hijos de los trabajadores formales a aquellos niños, niñas y adolescentes residentes en la República Argentina, que no tengan otra asignación y pertenezcan a grupos familiares que se encuentren desocupados o se desempeñen en la economía informal. Se otorga a todo ciudadano/a que cumpla con los requisitos básicos, estando su pago condicionado a la acreditación del cumplimiento de la escolaridad obligatoria, controles de salud y vacunación. Hacia fines de 2011, esta cobertura alcanzaba a alrededor de tres millones y medio de niños (Feijoó, 2013), instalándose dentro de las políticas de la seguridad social y asumiendo rasgos distintivos respecto de planes y programas asistenciales implementados en décadas anteriores (Feldfeber y Gluz, 2011).

Ubicada en el plano de extender un derecho y de garantizar el cumplimiento de otros, como los de la salud o la educación, la AUH prolonga una serie de políticas educativas y docentes previas cuyo eje central fue el de la inclusión escolar. Este argumento se condensó especialmente en la necesidad de lograr una incorporación con asistencia sostenida a las instituciones educativas, procurando ampliar la cobertura de niveles como el inicial o el secundario. Así, el discurso pedagógico oficial fortaleció el horizonte de la inclusión como una tarea central de la escuela y del trabajo docente, incluyéndola en los modos de razonar, interpretar y comprender sus desafíos y posibilidades. Este artículo se sitúa en explorar estos efectos a través del análisis de la construcción de posiciones docentes.

Los colectivos docentes de las tres escuelas que integraron el trabajo de campo comparten que la pérdida de matrícula, la repitencia y el desplazamiento del trabajo docente a una tarea de contención en el sentido asistencial del término son algunos de los elementos de las situaciones de desigualdad que el proyecto institucional que implementaron se propuso resolver. Se trataba de "reponer lo escolar", recuperar una escuela que consideraban perdida debido a que la institución en la que trabajaban había "tocado fondo". Por ello entendían la ausencia del trabajo de enseñanza de contenidos escolares y su aprendizaje por parte de los estudiantes. Para los docentes, la tarea docente se había reducido a la asistencialidad alimentaria y a infructuosos intentos por instalar normas compartidas, a intentar disminuir las situaciones de violencia escolar, a la "contención" en sentido estricto, y a intentar que, al menos, los alumnos concurrieran a la institución y permanecieran dentro de las aulas.

En ese marco, los docentes consideraban que era necesario llevar adelante alguna acción institucional que revirtiera esta serie de situaciones que las docentes sostenían que era necesario modificar. En algunos casos, ella se inscribía no sólo en la detención de la pérdida de matrícula sino también en la necesidad de que la escuela fuera un lugar para aprender. En efecto, 
las situaciones de discontinuidad en las experiencias formativas escolares de los niños o su ausencia componen un primer orden de dificultades a las que los colectivos de maestros intentarán proporcionar una alternativa. Por otro lado, estas premisas se fundan en la noción de que la escuela debe ser un lugar atractivo y estimulante para los estudiantes, dejando atrás su condición de lugar de "contención" para reposicionar a la "tarea pedagógica" en el lugar central de su razón de ser. La sensación predominante de los docentes es que les faltaban recursos para hacer de la escuela un lugar de trabajo "escolar".

Esta serie de preocupaciones, situaciones consideradas necesarias de ser modificadas y de ideas sobre la centralidad de la tarea pedagógica de enseñanza en la escuela no acontecen en el vacío. Integran un conjunto de producciones de significado y de movimientos del campo educativo que, desde las políticas educativas llevadas a cabo por el Estado Nacional, impulsaron la circulación de discursos que tuvieron efectos en los modos de pensar la docencia. En el período 2003-2015 es posible reconocer un conjunto de intervenciones desde el campo de la política que apuntaron a posicionar la enseñanza como tarea central del trabajo docente y que construyeron un argumento relativo a la inclusión educativa como una premisa estructurante (Vassiliades, 2014). El desarrollo de iniciativas tales como el Programa Integral para la Igualdad Educativa -implementado a partir de 2004 y dirigido a proporcionar recursos y condiciones pedagógicas a escuelas primarias-, la redefinición de temas en la agenda de la formación docente inicial y en ejercicio, el despliegue de programas vinculados a la cuestión de la inclusión como Conectar Igualdad, y la propia implementación de la Asignación Universal por Hijo, política que se afirmó sobre el argumento de la inclusión escolar de los estudiantes en edad escolar obligatoria, se ubican en la misma línea de movimientos. Como tales, contienen una serie de efectos de conocimiento (Popkewitz, 2000) sobre el trabajo docente, es decir, respecto de su concepción, interpretación, comprensión y sobre los modos de desarrollarlo y validarlo, que deben considerarse en la construcción de posiciones docentes en territorios de desigualdad.

"La escuela no es un lugar deseable o estimulante para los alumnos", tal una de las sentencias más contundentes enunciadas por los colectivos docentes de las tres escuelas, que operó como una de las condiciones de posibilidad de las iniciativas ensayadas en las tres escuelas que integraron la investigación. Las posiciones docentes presentan una serie de matices en relación a esta cuestión, en tanto oscilan entre promover la estadía regular de los alumnos en la escuela, y la promoción de la especificidad de la enseñanza como trabajo específicamente docente, como modo de habilitar otros horizontes además de una estadía escolar más prolongada. La posición docente se compone de estas miradas diversas, que pueden asumir ribetes paradójicos, y hasta suponer actitudes pocos satisfactorias para los mismos sujetos, aún cuando las sostengan. Es la construcción contradictoria, compleja y articulada 
de estos distintos "modos de pararse" frente a situaciones consideradas del orden de la desigualdad y por las que cabría hacer algo.

Las iniciativas institucionales elaboradas por los colectivos docentes de las escuelas que integraron el trabajo de campo ensayaron modificaciones en la organización del tiempo y el espacio. Las propuestas diseñadas presentan diferencias que dan cuenta de una diversidad de posiciones respecto de lo que es posible y deseable hacer con el formato escolar y habilitar desde él. En una de las escuelas, la cantidad de recreos fue reducida a dos, mientras que en la otra la grilla horaria escolar fue modificada, eliminándose tres de los cuatro recreos y extendiendo el restante a una duración de media hora, justo en la mitad de la jornada escolar. La jornada escolar pasó a estar organizada en dos módulos de cincuenta minutos, un recreo de treinta minutos, y otros dos módulos de cincuenta minutos. Estas nuevas pautas de organización del tiempo escolar constituyeron, para los docentes, un aporte sustantivo a poder desarrollar una forma de trabajo más sostenida y sistemática durante las clases:

"Los dos bloques largos de clases que el recreo corta en el medio hace que los chicos no se distraigan tanto. Y que se puedan concentrar en la clase, y comenzarla y terminarla, como la programaste. Que tal vez si tuviésemos recreo, los tres recreos que se suele tener ahi es como que te corta mucho hasta que salen, hasta que entran... Ahora se puede llegar a tener un clima de concentración en esta hora y cuarenta minutos y poder dar toda la clase prevista" (entrevista a docente, Escuela 1).

“...Y, está bien porque están contenidos en el recreo, no hay mala conducta, salvo lo normal. Pero antes eran tres recreos cortitos, pero no servían, en vez de jugar se peleaban, era terrible. Ahora por lo menos yo veo que están más contenidos" (entrevista a docente, Escuela 2).

Las modificaciones introducidas en el uso del tiempo escolar no remiten a una mera cuestión técnica sino que implican una discusión relativa a la configuración de este rasgo en cuanto a su organización -al desterrar las "horas" y los sucesivos recreos, y conformar "bloques" interrumpidos por un solo corte-, pero se afirman en una posición que contiene elementos de la propia historia del trabajo docente a la vez que también de los discursos contenidos en las iniciativas oficiales que los docentes reciben activamente, reforzándolas o reformulándolas.

El tiempo aparece como un recurso para regular y organizar las disposiciones de los alumnos. Lograr que "estén contenidos", "que no se desbanden", que "permanezcan concentrados", que "jueguen pero no peleen", que se "descarguen" en el recreo, constituyen propósitos que dan cuenta de una serie de definiciones que conservan pautas históricas de apelación del tiempo como herramienta de disciplinamiento en el contexto de una tarea moralizante (Alliaud, 1993; Birgin, 1999). En este sentido, en la modificación de algunos 
usos tradicionales del tiempo no parece haber la producción de algo enteramente novedoso sino más bien un verdadero "refinamiento de lo escolar": lograr alumnos más concentrados en las clases, en mejores condiciones para desarrollar las tareas y actividades propuestas por las docentes, y que utilicen el único recreo como momento de esparcimiento. Se trata de un tiempo escolar orientado a instalar una organización secuenciada de contenidos, momentos y ejercicios que apuntaban a que los sujetos aprendieran conforme a ciertas reglas (Pineau, 2001).

Sin embargo, las condiciones de posibilidad para ensayar usos diferentes del tiempo escolar no solamente se hallan en la construcción histórica del trabajo de enseñar en Argentina y en la región. Estas reformulaciones implican también una apropiación de las pautas para el trabajo docente que integran las regulaciones de la política educativa del Estado provincial. Como he analizado en trabajos previos (Vassiliades, 2014), ellas se organizan en torno de la idea de inclusión escolar y plantean la necesidad de reposicionar la "centralidad de la enseñanza" como eje de la tarea docente. Entre los caminos que los documentos de política educativa plantean para ello, se destaca la posibilidad de ensayar alternativas a la organización institucional, aunque no se explicita la sugerencia de modificar el modo de plantear el tiempo escolar. Los modos en que las desarrollan los docentes de las tres escuelas parecen dar cuenta de una manera particular de apropiarse de los significados puestos a circular por el discurso pedagógico oficial.

Las iniciativas desarrolladas por los colectivos docentes prevén, asimismo, la reorganización y redefinición de los espacios institucionales y del tránsito por ellos de los sujetos escolares. Las iniciativas no son homogéneas y dan cuenta de propuestas diversas respecto de qué hacer con el formato escolar. En una de las escuelas se instituyó el trabajo por áreas en segundo ciclo, de cuarto a sexto grado, dando como resultado que cada grado está a cargo de tres docentes, aunque sin modificar la organización de espacios escolares vigente. Los maestros y directivos señalan que esta iniciativa, impulsada de manera conjunta luego de una capacitación organizada por el FOPIIE -el Programa de Fortalecimiento del PIIE-, obedece al "agotamiento" que le producía a un solo docente estar a cargo de un grupo y tener que lidiar con "situaciones bastante delicadas de conducta". La idea principal consistía en que la responsabilidad de hacerlo no cayera sobre un solo docente sino que fuera compartida.

"Fue una propuesta de los directivos, que vinieron y lo plantearon en una reunión. Porque por ahí los grados, si bien no son numerosos, tenés un nivel de conducta en los pibes que se te hace insoportable, te agota. Entonces, a ver si podíamos por ahí poder sobrellevar las cosas y que no se recargue todo sobre un solo docente" (entrevista a docente, Escuela 3). 
En otra de las escuelas, si bien aún no se plasmó la idea de trabajar por áreas, sí se ha definido que aquellos maestros con más experiencia -"los mejores", según los docentes entrevistados- sean quienes trabajen en primer ciclo y que, comenzando desde primer grado, "pasen" de nivel junto con su grupo para poder hacer un mejor seguimiento de los alumnos. Los posicionamientos respecto del trabajo por áreas no son homogéneos. Fuera de los marcos formales de una entrevista o de una jornada institucional registrada para el trabajo de campo, conversaciones informales sostenidas con algunos docentes en los pasillos de las instituciones dan cuenta de que hay quienes prefieren no trabajar por áreas porque sienten que ello les hace perder el status de maestros "de grado". Algunos prefieren trabajar en un solo aula, "entrar, cerrar la puerta y saber que estoy frente a mi grado, mi grupo", y que esa condición es la que, justamente, les permite tener un mayor y mejor manejo de las situaciones conflictivas. Por otra parte, hay docentes que resaltan que, organizando el trabajo por áreas, tienen mayores posibilidades de visualizar los avances y dificultades de sus alumnos en las diversas áreas, a la vez que ellos tienen otros adultos referentes en lugar de uno solo. Esta pluralidad de miradas es valorada frente a la soledad que suele caracterizar al trabajo docente:

“¿Sabes qué pasa? Uno como docente dentro del grado está muy solo y de golpe y de repente tenés a otras personas que te acompañan. Y tener tres miradas diferentes sobre un determinado chico es importante. $Y$ para los padres también es importante. Porque los padres a veces tienen la idea que uno viene ya con la idea 'Ah, él lo rechaza a mi hijo' Sin embargo, hay tres personas que le están diciendo 'Mira, le falta esto, aquello, que ponga un poco más de atención' es como que los padres recién ahí toman conciencia" (Entrevista a docente, Escuela 1).

"Ésta es la diferencia, que los chicos puedan tener un acercamiento mayor y la manera de observar al chico es diferente. ¿Por qué? Porque somos tres observándolo, y no una. Yo tengo un pibe en sexto que trabaja en matemática de determinada manera, en lengua puede trabajar de otra y en ciencias puede trabajar absolutamente de otra manera. La visión que tenemos las tres del pibe es diferente y no está condicionado por una sola mirada" (entrevista a docente, Escuela 2).

Como puede observarse, la pluralidad de miradas es también ponderada por permitir mejorar la "observación" sobre los alumnos y lograr en ellos las conductas deseadas. Al tiempo que se habilita que los estudiantes trabajen de modo diferente en las diversas materias, se asocia el hecho de que los ojos de tres maestras estén sobre los alumnos a la posibilidad de que ellos desarrollen otras actitudes y disposiciones, como el prestar "un poco más de atención", el aceptar a las maestras "como referentes" y el logro de "cosas más positivas". Estas estrategias vinculan la llamada "reposición del escolar" al hecho de que 
los pibes se vuelvan los alumnos esperados dentro de las expectativas de las docentes.

En el caso de otra de las escuelas, las modificaciones implementadas avanzan no sólo en la redefinición del trabajo y su organización en áreas, sino también en la reformulación de los espacios escolares. En la propuesta elaborada por el colectivo docente de la institución, las tradicionales aulas dejan paso a las "aulas-taller", cuya característica principal es la de ser lugares en donde se trabaja específicamente sobre una de las áreas. Así, hay aulas-taller de matemática, de prácticas del lenguaje y de ciencias -que incluye a las ciencias naturales y a las ciencias sociales. Las "aulas-taller", entonces, ya no se homologan a los grados -como las anteriores aulas- ni son espacios en donde los alumnos permanecen durante toda la jornada, sino que éstos circulan por ellas. De este modo, cada grado transita por dos o tres aulas-taller por día. Los docentes ya no son "maestros de grado", sino que están a cargo de cada una de las áreas durante un ciclo. Esta definición pone en tensión un modo histórico de pensar el trabajo docente en las instituciones escolares -la idea de que un grupo de alumnos pertenece a un maestro y viceversa-, que se articula con modos colectivos de pensar la tarea de enseñar y la resolución de problemas pedagógicos. Sobre ello me detendré a continuación.

\section{Incluir y construir colectivamente la tarea pedagógica: "los pibes son de todas"}

En las tres escuelas los colectivos docentes asocian las iniciativas implementadas al "agotamiento" que le producía a un solo docente estar a cargo de un grupo en las coordenadas de trabajo anteriores y tener que lidiar con "situaciones bastante delicadas de conducta". La idea principal consistía en que la responsabilidad de hacerlo no cayera sobre un solo docente sino que fuera compartida. A simple vista, esta suerte de "socialización" de los problemas escolares podría suponer un intento por alivianar la responsabilidad referida al trabajo que deben desarrollar como docentes. Sin embargo, a partir de los datos construidos a lo largo del trabajo de campo, parece implicar una necesidad de tornar colectivos ciertos procesos que, llevados a cabo de manera individual, afectarían profundamente el trabajo en la escuela.

Las nuevas pautas de organización del trabajo docente en las tres escuelas parecen estar acompañadas de estrategias "informales" que apuntan a lograr el fortalecimiento de una tarea colectiva en torno de un proyecto específico. Por ejemplo, los recreos suelen ser ocasiones en donde las maestras hilvanan la interdisciplinariedad de su trabajo en la escuela, desarrollando intercambios respecto de los aportes que podría realizar cada área al proyecto. Ello se plasma en conversaciones donde están presentes las consultas, sugerencias y solicitudes entre colegas, referidos al trabajo sobre un tema en particular. 
Este intercambio expresa también la preocupación, manifestada recurrentemente por las maestras, de que la organización en áreas no fragmente el trabajo de enseñanza sino que, al contrario, se vuelva posible desarrollar el trabajo colectivamente. La tarea docente, en particular aquélla desarrollada habitualmente en un solo grado, puertas adentro del salón de clase, suele constituir una actividad "en soledad" con escasas condiciones institucionales y laborales para el intercambio y el trabajo colectivo. Por lo general, los únicos momentos de encuentro de maestros suelen ser las llamadas "horas especiales"-aquellos espacios en donde los alumnos tienen asignaturas como inglés, música, educación física o plástica, que no suelen contar como materias "básicas"-, dado que allí quedan disponibles para desarrollar su trabajo fuera del salón de clase.

La "colectivización" de algunos procesos implican operaciones que las maestras llevan a cabo para poder enseñar. La propuesta del trabajo por áreas ensaya una posible superación del "individualismo áulico" promovido por las condiciones laborales docentes tradicionales, habilitando otras coordenadas institucionales para el trabajo de enseñanza. En la búsqueda de estas alternativas pareciera haber un intento de sostener el proyecto de educar desde inscripciones más colectivas. En este marco, se ensayan resoluciones a cuestiones que los docentes atribuyen al desarrollo de políticas como la AUH: una asistencia de los alumnos más sostenida que antes, una regularidad en la concurrencia que es interrumpida por lapsos más breves, una presencia mayor en las instituciones escolares del grupo de estudiantes, la posibilidad -que, para las docentes, habilitaría la $\mathrm{AUH}$ - de contar con más útiles o de alumnos con una vestimenta en mejores condiciones, y una progresiva mayor cercanía de las familias respecto de la actividad escolar.

En este marco, una inscripción más de estas construcciones de sentido en torno de la docencia es la idea de que los alumnos son de todas, y no de alguna maestra en particular. Esta idea está estimulada por la circulación de alumnos por las aulas y por el hecho de que el trabajo docente a lo largo de un ciclo favorece el hecho de que varias maestras se encuentren con más de un grupo de alumnos. La idea de que "los pibes son de todas" rompe con una de las consecuencias principales de un rasgo central de la gramática escolar, que postula la existencia de grupos de edades a cargo de un maestro, que por lo general es el único en la escuela que conoce a sus alumnos, además del director. En el caso del trabajo de campo, la idea de que los alumnos son de todos los docentes instala una noción de lo común para la experiencia escolar que se inscribe en las posiciones docentes frente a la desigualdad que aquí estoy analizando.

"Los pibes son de todas" tiene expresiones en diversas posiciones. En algunos casos, supone que la enseñanza y el seguimiento de los aprendizajes constituyen una responsabilidad colectiva a asumir por el conjunto de los 
Revista de la Escuela de Ciencias de la Educación, año 13, nRo. 12, vol. 1, enero a junio de 2017. Páginas 7996. ISSN 1851-6297 - ISSN 2362-3349 (EN LiNEA). Trabajo docente e inclusión educativa en tiempos de la asignación universal por hijo: posiciones, experiencias y sentidos en disputa. ALEJANDRO VASSILIADES

docentes de la institución. En este marco, son habituales los intercambios respecto de la progresión en los contenidos de los estudiantes, las dificultades que surgen, la manera de trabajar articuladamente y las posibles resoluciones que estos problemas podrían tener:

"En el recreo de media mañana, cinco docentes se encuentran sentados en torno de la mesa que hace las veces de kiosco escolar para recaudar fondos para comprar un proyector. Una de ellas, de Matemática de primer ciclo, le comenta a otra, de Prácticas del Lenguaje acerca de las dificultades de dos alumnas para leer consignas, solicitando su asistencia. La docente de Matemática de segundo ciclo interviene como si conociera a las alumnas de las que están hablando, dando una sugerencia referida al trabajo oral en los problemas matemáticos. La docente de Prácticas del Lenguaje propone trabajar con las consignas de esa materia en su clase. Otra docente, de Ciencias, también comenta acerca de algunas dificultades para la lectura en su materia. En estos intercambios es imposible decir de quién son los alumnos, o de quién no lo son, ya que pareciera ser que la escuela es un gran grupo de alumnos a cargo de las seis maestras de grado" (nota de campo, Escuela 1).

"Acá no hay alumnos tuyos y alumnos míos. Los alumnos son de todos nosotros. Por lo general si hay algún problema tratamos de charlarlo y ver todos juntos cómo lo resolvemos, no hay esa cosa individual de que te encerrás en el aula y el grupo es tuyo... al contrario, ¿entendés?. Si bien cada uno tiene su grupo, los temas que surgen los vamos viendo entre todos" (entrevista a docente, Escuela 2).

Esta responsabilidad compartida no sólo expresa que "los pibes son de todas", sino que las docentes también son de todos los estudiantes. En algunos casos, se asocia este compromiso al cuidado de los alumnos, el afecto y la escucha. Las maestras conocen los nombres y las historias de los alumnos de grados distintos a los suyos -y no solamente de aquellos estudiantes que tienen o alguna vez tuvieron. Asimismo, los niños se vinculan con cada maestra como si fuera "su" docente, aparentemente sin distinción de grado:

"Nosotras conocemos las historias de todos; es raro que vos tengas un alumno que los demás maestros no sepan sobre su familia, las cosas que le pasan, si está viniendo o no viniendo a la escuela, si se enfermó o si a los padres les pasó algo" (entrevista a docente, Escuela 2).

"Durante el recreo, algunas alumnas que deben ser de quinto o sexto grado se acercan a saludar y abrazar a las maestras allí presentes, que son tanto de primero como de segundo ciclo. Apenas se van, llega la directora, quien les hace un comentario respecto de la desaparición de una hermana 
de un alumno del hogar. Todas las maestras intercambian entre sí, como si conocieran la historia de cerca. Una de ellas relata que, en una ocasión anterior, la niña no había desaparecido sino que un familiar suyo la estaba "vendiendo" en un asentamiento cercano. Otra asiente diciendo que estaba al tanto, mientras que una tercera se manifiesta horrorizada. Una cuarta docente comenta que había estado hablando con el alumno en cuestión días atrás porque lo había notado muy extraño" (nota de campo, Escuela 1).

Estas manifestaciones están emparentadas con el modo en que las maestras conciben y asumen la responsabilidad adulta frente a sus alumnos, un aspecto nodal de la construcción de la autoridad docente (Birgin, 2006). En algunos casos se realizan consideraciones respecto de que tal es el trabajo que como docente debe realizarse, y el que corresponde hacer. En otros, esa asunción se sustenta en el planteo de que los problemas, si existen, no son de los niños sino de los adultos. Esta posición se afirma en la idea de que es responsabilidad de "los grandes" -la familia, los docentes y directivos de una escuela- lidiar con y tratar de resolver las dificultades que se presentan en la cotidianeidad con los niños, y que por ello cabe preservarlos de que éstos tengan que enfrentarse a esta tarea. Algunos docentes incluso afirman que se trata de un derecho que los niños y niñas tienen de no ser cargados con una responsabilidad que no les corresponde. Otros, por su parte, revisan y reinterpretan lo que acontece cotidianamente en las instituciones a la luz de estas premisas, sosteniendo una posición más tradicional respecto de cuáles son los alumnos que un maestro debe tener a su cargo:

"Yo estoy convencida de que el problema es del adulto, no del pibe. Y muchos chicos que con 17, 18, terminan la escuela de adultos hoy por hoy, no es el fracaso del alumno, es el fracaso del adulto. Es el fracaso del adulto. Hay que tomar la responsabilidad, yo digo 'a ver, vos que estas en la escuela, estas a cargo de un grado, tu responsabilidad ahora es tu grupo"” (entrevista a docente, Escuela 3).

Como puede verse, las asunciones respecto de la diferencia que debe sostenerse con los alumnos no son homogéneas. "Los pibes son de todas" constituye una premisa que condensa las significaciones en torno de la posición ética que desarrollan las docentes en torno de sus pibes y el modo en que se plantean el trabajo con ellos. Ello implica el conocimiento de sus historias, el cuidado cotidiano, la preocupación tornada explícita, el acercamiento para saber acerca de sus problemas, y también una responsabilidad relativa a proporcionarles herramientas para que los aprendizajes acontezcan.

Aún sosteniendo diversos modos de vincularse con sus alumnos, los docentes afirman que hay un compromiso mayor por parte de sus alumnos respecto del trabajo escolar y que ello tiene que ver con la apuesta que han desarrollado y sostenido a que ellos aprendan. Esta posición se expresa en 
la apertura de una serie de espacios institucionales y la habilitación de trayectorias escolares específicas de acuerdo a las necesidades de aprendizaje de sus estudiantes. Los maestros reconocen que en estas modificaciones han podido ver que sus alumnos, aquéllos que antes del proyecto "no podían", y con los que era "imposible trabajar", pueden aprender y vislumbrar que otros futuros estén habilitados para ellos.

Las docentes también "pueden". En las situaciones de clase registradas, y aunque con matices, fue posible observar que la situación de precariedad laboral y material no constituyen un obstáculo insalvable para la enseñanza como vía de ofrecer lo que las maestras consideran lo más importante que tiene para dar la escuela a sus alumnos, en línea con los hallazgos de investigaciones previas (Redondo y Thisted, 1999; Redondo, 2004). Las pésimas condiciones edilicias, los magros salarios, la falta de recursos -muchas veces suplidos por los propios docentes- no se tornaron un límite imposible de franquear para desarrollar la tarea de enseñar. El esfuerzo por enseñar los conocimientos escolares y por democratizar el acceso a ellos constituye una de las iniciativas más importantes respecto de lo que las maestras consideran que cabe hacer frente a situaciones que conciben como de desigualdad. Estas iniciativas se basa en que dicho acceso tiene carácter emancipatorio y en la idea de que todos los alumnos pueden ser aprender. Esta idea, ligada a la universalización de la vigencia del derecho a la educación, también encuentra su condición de posibilidad en su circulación previa en la discursividad oficial de la que las políticas educativas del período 2003-2015 dan cuenta.

\section{A modo de cierre: efectos del imperativo de la inclusión}

A lo largo de este trabajo he intentado dar cuenta de los modos en que sujetos docentes de tres escuelas primarias públicas de la provincia de Buenos Aires configuran sentidos acerca de su trabajo que condensan modos diversos de procesar el mandato de la inclusión educativa. Como he intentado mostrar, una posibilidad analítica abierta por la categoría de posición docente respecto de otros modos de referirse al trabajo de enseñar (por ejemplo, los que lo enuncian como un "rol" o una "función") es la de haber podido considerar la heterogeneidad de modos en que los elementos que la integran se despliegan y la imposibilidad de determinar estas definiciones a priori. Asimismo, la categoría de posición docente permitió también considerar las dimensiones pedagógicas del trabajo de enseñar.

Los modos distintos de pensar el trabajo de enseñar, el tiempo y el espacio escolares, y los problemas relativos a la desigualdad educativa se inscriben en la línea de intentar producir alternativas al formato escolar tradicional, construyendo otras formas de albergar a las nuevas generaciones, de vincularse con ellas y de intentar desarrollar el trabajo de transmisión cultural. Esas alternativas también posibilitaron otro abordaje de las situaciones de desigual- 
dad social y educativa, que en los modos de trabajo tradicional suelen situar en el lugar del "fracaso escolar" a quienes viven en condiciones de pobreza y son pibes que trabajan en los trenes y en las esquinas, cuidan a sus hermanos o están internados en hogares, y que atraviesan situaciones de repitencia, sobreedad y expulsión escolar. Los modos de pensar otros posibles formatos escolares implican una interrupción de esa serie y el armado de otra diferente en torno de la enseñanza de los contenidos escolares.

En este movimiento parecen tornarse visibles una serie de temas y problemas que permanecían soslayados antes de la implementación de las iniciativas institucionales: qué configuración de tiempos escolares darse, qué distribución de espacios escolares habilita mejores aprendizajes y cómo organizar el trabajo docente de manera tal de potenciar la propuesta contenida en los diseños curriculares. Estas definiciones constituyeron modos específicos de abordar situaciones que, desde las posiciones docentes construidas, configuraban situaciones de desigualdad educativa. Una inscripción del compromiso sostenido desde las posiciones docentes tiene que ver con el desarrollo de formas de autoridad adulta que implican modos de entender y desplegar la responsabilidad por las nuevas generaciones. "Los pibes son de todas" es una idea que condensa el modo en que las maestras sumaron especificidades propias e invenciones desarrolladas al calor de la puesta en marcha de las innovaciones institucionales, sobre la base de modos diversos de procesar la idea de inclusión.

Lo señalado hasta aquí hace posible entablar una discusión con aquellas perspectivas fundadas en el "déficit" o la "imposibilidad" de los maestros, desde las que se pretende decir todo lo que los docentes "no son", "no tienen" y "no pueden", y brindar algunas recetas para colmar ese supuesto vacío que esas propias perspectivas construyen. Ello supone una perspectiva más atenta a no producir como ausentes un conjunto de experiencias que, llenas de heterogeneidades, muestran que los y las docentes hacen, pueden, enseñan, desafían los límites "del contexto", construyen formas de autoridad adulta y se hacen cargo de la tarea de pasarles algo de eso que consideran valioso a las nuevas generaciones. Tornar visibles los modos en que cotidianamente los maestros y maestras se apropian, redefinen y disputan las regulaciones de su trabajo y los sentidos en torno del imperativo de la inclusión podría resultar un aporte para desarrollar comprensiones acerca de los vínculos entre trabajo docente, escolarización y desigualdad, y respecto de los efectos pedagógicos de las políticas educativas y docentes desarrolladas en los últimos años.

\section{Notas Bibliográficas}

(1) Este artículo presenta resultados de la investigación desarrollada en el marco de mi tesis de doctorado "Regulaciones del trabajo de enseñar en la provincia de Buenos Aires: posiciones docentes frente a la desigualdad social y educativa", dirigida por la Dra. 
Revista de la Escuela de Ciencias de la Educación, año 13, nRo. 12, vol. 1, enero a junio de 2017. Páginas 7996. ISSN 1851-6297 - ISSN 2362-3349 (EN LiNEA). Trabajo docente e inclusión educativa en tiempos de

la asignación universal por hijo: posiciones, experiencias y sentidos en disputa. ALEJANDRO VASSILIADES

Myriam Southwell y realizada en la Facultad de Filosofía y Letras de la Universidad de Buenos Aires, y del trabajo en curso en el marco del proyecto "El trabajo de enseñar en tiempos de la Asignación Universal por Hijo: posiciones docentes frente a las desigualdades sociales y educativas", que actualmente llevo adelante en el marco de la Carrera de Investigador Científico y Tecnológico del CONICET.

\section{Referencias bibliográficas}

- Alliaud, A. (1993). Los maestros y su historia: los orígenes del magisterio argentino. Buenos Aires: Centro Editor de América Latina.

- Birgin, A. (1999). El trabajo de enseñar. Entre la vocación y el mercado: las nuevas reglas del juego. Buenos Aires: Troquel.

- Birgin, A. (2006). Pensar la formación de los docentes en nuestro tiempo. En F. Terigi (comp.) Diez miradas sobre la escuela primaria (pp. 267-294). Buenos Aires: Fundación OSDE - Siglo XXI Editores.

- Buenfil Burgos, R. N. (2007). Introducción. En P. Padierna Jiménez y R. Mariñez (coords.) Educación y comunicación. Tejidos desde el Análisis Político del Discurso (pp. 15-35). México: Casa Juan Pablos - Programa de Análisis Político del Discurso e Investigación.

- Dussel, I. (2004). Inclusión y exclusión en la escuela moderna argentina: una perspectiva postestructuralista. Cadernos de Pesquisa, Vol. 34, N 122, 305-335.

- Feijoó, M. (2013). Asignación Universal y política educativa. Le Monde Diplomatique. Suplemento UNIPE. Edición NN 168. Buenos Aires: Universidad Pedagógica, 1-4.

- Feldfeber, M.y Gluz, N. (2011). Las políticas educativas en Argentina: herencias de los '90, contradicciones y tendencias de nuevo signo. Educaçao e Sociedade. Volume 32. Campinas: CEDES, 339-356.

- Laclau, E. (1993). Emancipación y diferencia. Buenos Aires: Ariel.

- Pineau, P. (2001). ¿Por qué triunfó la escuela?, o la modernidad dijo: 'Esto es educación' y la escuela respondió: 'Yo me ocupo'. En P. Pineau, I. Dussel y M. Caruso, La escuela como máquina de educar (pp. 21-52). Buenos Aires: Paidós.

- Popkewitz, T. (2000). Sociología política de las reformas educativas. Madrid: Morata.

- Redondo, P. (2004). Escuelas y pobreza. Entre el desasosiego y la obstinación, Buenos Aires: Paidós.

- Redondo, P. y Thisted, S. (1999). Las escuelas 'en los márgenes`. Realidades y futuros. En AA.VV. En los límites de la educación. Niños y jóvenes del fin de siglo (pp. 21-52). Buenos Aires: Homo Sapiens.

- Southwell, M. (2007). Profesionalización docente al término del siglo XX: políticas y nominaciones producidas por organismos internacionales. Anuario de Historia de la Educación. Nº 8. Buenos Aires: Prometeo, 261-285.

- Southwell, M. (2009). Docencia, tradiciones y nuevos desafíos en el escenario contemporáneo. En J. Yuni, José (comp.) La formación docente. Complejidad y ausencias , (pp. 169-199). Córdoba: Encuentro Grupo Editor.

- Southwell, M. (2012) (comp.). Entre generaciones. Exploraciones sobre educación, cultura e instituciones. Buenos Aires: FLACSO - Homo Sapiens.

- Southwell, M. y Vassiliades, A. (2014). El concepto de posición docente: notas 
Revista de la Escuela de Ciencias de la Educación, año 13, nRo. 12, vol. 1, enero a junio de 2017. Páginas 7996. ISSN 1851-6297 - ISSN 2362-3349 (EN LiNEA). Trabajo docente e inclusión educativa en tiempos de la asignación universal por hijo: posiciones, experiencias y sentidos en disputa. ALEJANDRO VASSILIADES

conceptuales y metodológicas. Revista Educación, Lenguaje y Sociedad. Volumen XI, № 11. General Pico: Instituto para el Estudio de la Educación, el Lenguaje y la Sociedad, 163-187.

- Vassiliades, A. (2012). Regulaciones del trabajo de enseñar en la provincia de Buenos Aires: posiciones docentes frente a la desigualdad social y educativa. Tesis de Doctorado. Buenos Aires: Facultad de Filosofía y Letras de la Universidad de Buenos Aires.

- Vassiliades, A. (2014). El discurso pedagógico oficial en Argentina (2003-2013): trabajo docente e igualdad. Cadernos de Pesquisa. V. 44, n 154. São Paulo: Fundação Carlos Chagas, 1012-1027. 